\title{
Role of Causality in the Evaluation of Coherence and Transfer Function between Heart Period and Systolic Pressure in Humans
}

\author{
L Faes $^{1}$, A Porta $^{2}$, R Antolini ${ }^{1}$, G Nollo $^{1}$ \\ ${ }^{1}$ Department of Physics, University of Trento, Trento, Italy \\ ${ }^{2}$ Dipartimento di Scienze Precliniche, Milano, Italy
}

\begin{abstract}
To elicit the effects of considering causality in the study of the interactions between RR interval and systolic pressure (SP) variability, the traditional noncausal crossspectral analysis was compared with a causal method able to separate the two arms of the RR-SP regulatory loop. Estimates of coherence $(K)$ and causal coherences from $S P$ to $R R$ (Ksr) and from $R R$ to $S P(K r s)$, and of noncausal $(G)$ and causal (Gsr) baroreflex gain were evaluated at $0.1 \mathrm{~Hz}$ in 10 healthy young subjects in the supine position and after head-up tilt. While $K$ was high in both conditions, at rest Ksr was significantly lower than Krs. After tilt, Ksr increased and Krs decreased significantly. With respect to G, Gsr was significantly lower at rest and comparable after tilt. Thus, in the supine position the prevalence of non-baroreflex coupling mechanisms led the traditional approach to overestimate the baroreflex gain, while the tilt-induced activation of the baroreflex control made similar the gain estimates.
\end{abstract}

\section{Introduction}

The study of the interactions between the heart period (RR interval) and the systolic pressure (SP) is commonly performed by means of cross-spectral analysis, a classical frequency-domain tool which provides useful information about the strength of the coupling between the cardiovascular variables (through the coherence function), and is also used to provide spontaneous estimates of the baroreflex gain (trough the modulus of the RR-SP transfer function) $[1,2]$.

The correct application of cross-spectral analysis requires the existence of a linear open loop interaction between the two investigated variability series. While the linearity of the interaction may be considered as valid for small RR and SP fluctuations around the working point, the open loop hypothesis can be hardly assumed in cardiovascular variability analysis. Indeed, in many experimental conditions RR interval and SP are likely to interact in a closed loop, as a result of the contemporaneous presence of the feedback baroreflex mechanism and of feedforward mechanisms operating in the opposite direction from RR to SP [3]. To overcome this limitation, we have recently proposed a causal approach for estimating the coherence [4] and the transfer function [5] along a given input-output direction, thus forcing cross-spectral analysis to be performed in open loop conditions even when significant interactions are present in the opposite direction.

The aim of this study was to assess, in different experimental conditions, the importance of accounting for causality in the estimation of the coherence between RR interval and SP and of the cross-spectral measure of the baroreflex gain. To this end, traditional and causal estimates of the coherence and the transfer function from SP to RR were compared in healthy young subjects in the resting supine position and after head-up tilt.

\section{Methods}

\subsection{Cross-spectral analysis of $R R$ interval and SP interactions}

The cross-spectral analysis of the variability of RR interval ( $r$ series) and SP ( $s$ series) is performed starting from the auto- and cross-spectral density functions $\left(P_{r}(f), P_{s}(f)\right.$, and $\left.P_{s r}(f)\right)$ of the two series. The coherence function

$\gamma_{s r}^{2}(f)=\frac{\left|P_{s r}(f)\right|^{2}}{P_{s}(f) P_{r}(f)}$

measures in the frequency domain the strength of the linear coupling between $r$ and $s$, and ranges from 0 (complete uncoupling) to 1 (full coupling).

The transfer function describes the transfer of power from one series to the other, according to a predefined input-output direction. When the baroreflex transfer function is considered (i.e., with $s$ as input and $r$ as output), the gain estimate based on the spontaneous variability of RR interval and $\mathrm{SP}$ is given by 
$G_{s r}(f)=\frac{\left|P_{s r}(f)\right|}{P_{s}(f)}$

\subsection{Parametric estimation of spectral density functions}

According to parametric cross-spectral analysis [6], the coherence and gain functions of (1) and (2) are estimated by taking the spectral density functions obtained from the fit of a bivariate autoregressive (AR) model on the available realizations of $s$ and $r$. The bivariate AR process representing the interactions between $s$ and $r$ is

$$
\begin{aligned}
& s(t)=\sum_{k=1}^{p} a_{11}(k) s(t-k)+\sum_{k=1}^{p} a_{12}(k) r(t-k)+w_{1}(t) \\
& r(t)=\sum_{k=0}^{p} a_{21}(k) s(t-k)+\sum_{k=1}^{p} a_{22}(k) r(t-k)+w_{2}(t)
\end{aligned}
$$

where $w_{1}$ and $w_{2}$ are uncorrelated zero-mean white noises with variance $\sigma_{1}{ }^{2}$ and $\sigma_{2}{ }^{2}$ and $p$ is the model order. Notice that $a_{11}(0)=a_{22}(0)=0$ to guarantee causality, and that $a_{12}(0)=0, a_{21}(0) \neq 0$ to guarantee the identifiability of the bivariate process [6].

After transforming (3) in the frequency domain, evidencing the transfer function matrix from the white noise inputs to the output series $r$ and $s$, and calculating the power spectral density matrix, the auto-spectra and the cross-spectrum result

$$
\begin{aligned}
& \left.P_{s}(f)=\left.|\Delta(f)|^{2}|| A_{22}(f)\right|^{2} \sigma_{1}^{2}+\left|A_{12}(f)\right|^{2} \sigma_{2}^{2}\right) \\
& \left.P_{r}(f)=\left.|\Delta(f)|^{2}|| A_{21}(f)\right|^{2} \sigma_{1}^{2}+\left|A_{11}(f)\right|^{2} \sigma_{2}^{2}\right) \\
& P_{s r}(f)=|\Delta(f)|^{2}\left(A_{22}(f) A_{21}^{*}(f) \sigma_{1}^{2}+A_{12}(f) A_{11}^{*}(f) \sigma_{2}^{2}\right)
\end{aligned}
$$

with $\Delta(f)=\left[\left(A_{11}(f) A_{22}(f)-A_{12}(f) A_{21}(f)\right]^{-1}\right.$, where

$$
A_{l m}(f)=\delta_{l m}-\sum_{k} a_{l m}(k) e^{-j 2 \pi f k},
$$

$l, m=1,2 ; \delta_{l m}=1$ for $l=m, \delta_{l m}=0$ for $l \neq m$.

\subsection{Causal cross-spectral analysis}

The autospectra and the cross-spectrum of the series $s$ and $r$, and consequently the coherence and transfer function values, are affected both by the coefficients describing the self-influences on the two series $\left(a_{11}\right.$ and $a_{22}$ ) and by the coefficients describing the mutual influences from one series to the other $\left(a_{12}\right.$ and $\left.a_{21}\right)$. As a consequence, the estimated coherence function is not able to distinguish whether a detected degree of coupling comes from feedback influences from $s$ to $r$ or from feedforward interactions from $r$ to $s$, while the baroreflex transfer function may be affected by the presence of feedforward reverse influences from $r$ to $s$.

To overcome these limitations, a causal cross-spectral analysis can be performed, after bivariate AR identification, by setting to zero the coefficients describing the mutual influences in the direction opposite to that under analysis [4]. In this way, the causal coherences from $s$ to $r$ and from $r$ to $s$ are defined as [4]

$$
\begin{aligned}
& \gamma_{s \rightarrow r}^{2}(f)=\left.\gamma_{s r}^{2}(f)\right|_{A_{12}(f)=0} \\
& \gamma_{r \rightarrow s}^{2}(f)=\left.\gamma_{s r}^{2}(f)\right|_{A_{21}(f)=0}
\end{aligned}
$$

while a causal estimate of the baroreflex gain is [5]

$$
G_{s \rightarrow r}(f)=\left.G_{s r}(f)\right|_{A_{12}}(f)=0
$$

\subsection{Experimental protocol and data analysis}

Ten healthy young subjects (age 25 \pm 3 years) were considered for the analysis. RR interval and SP series were taken from the ECG and the photopletismographic arterial pressure signal with subjects in the supine resting position and after passive $60^{\circ}$ head-up tilt.

Before performing cross-spectral analysis, the variability series were windowed to 300 samples, cleaned up from artifacts, and detrended to fulfill stationarity criteria. Starting from each pair of $r$ and $s$ series, the bivariate AR model (3) was identified by the least squares approach described in [6]. The model order was selected according to the Akaike's criterion for multivariate processes [7]. The estimated model coefficients were then used to calculate the coherence and transfer function gain using both noncausal and causal approaches. Finally, representative coherence and gain values were obtained by sampling the corresponding functions at the frequency of the maximum coupling inside the low-frequency band of the spectrum (LF, 0.03-0.15 Hz) [3].

The significance of the coupling and of the causal coupling between two series was assessed by means of a statistical approach based on surrogate data [8]. This method produces a zero level function which is used as threshold to assess at each frequency whether a given coherence or causal coherence value indicates a statistically significant degree of coupling or not.

\section{Results}

A representative example of the calculation of the coherence between RR interval and SP in a healthy young 
subject by noncausal and causal approaches is depicted in Fig. 1. Whereas the LF values of the traditional coherence are very high in both supine and upright positions, the causal coupling analysis reveals a different pattern of interaction between RR and SP. Indeed, at rest the causal link is significant from RR to SP but not from SP to RR $\left(\gamma_{s \rightarrow r}^{2}\right.$ is below the zero coherence threshold), while the opposite situation $\left(\gamma_{s \rightarrow r}^{2}\right.$ significant and $\gamma_{r \rightarrow s}^{2}$ non significant) is observed after head-up tilt. (a)
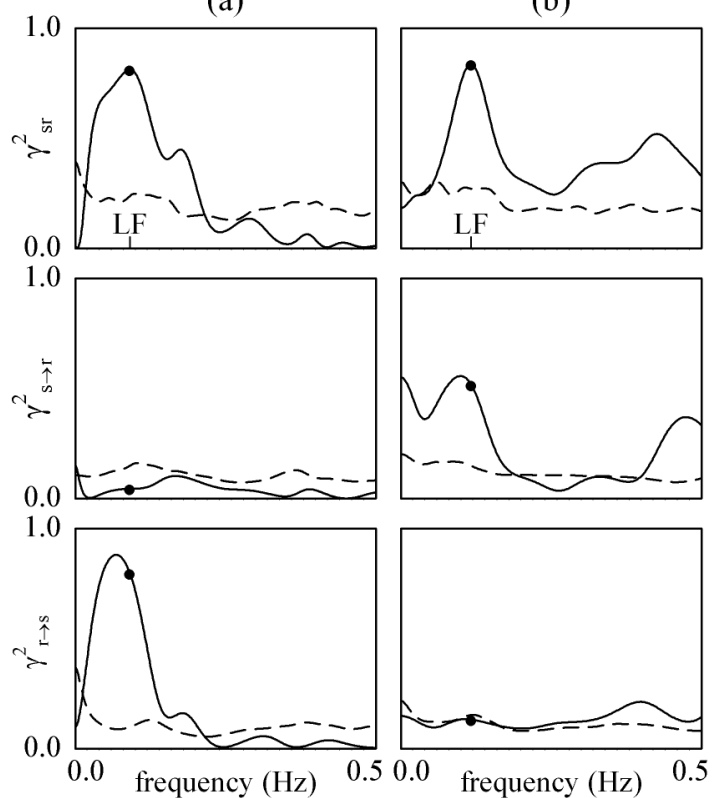

Figure 1. Example of calculation of the coherence $\left(\gamma_{s r}^{2}\right)$ and of the causal coherences from $s$ to $r\left(\gamma_{s \rightarrow r}^{2}\right)$ and from $r$ to $s\left(\gamma_{r \rightarrow s}^{2}\right)$ for a subject at rest (a) and after head-up tilt (b). Dashed lines represent the zero coherence thresholds. Filled circles indicate the functions sampled at the maximum coupling inside the low frequency (LF) band.

The pattern of interaction between RR and SP shown in Fig. 1 was confirmed by averaging the coherence values over the 10 analyzed subjects. Indeed, as shown in Fig. 2, high values of the coherence found both at rest and after tilt corresponded to a prevalence of the feedforward interactions from $r$ to $s$ at rest, and of the feedback effects from $s$ to $r$ after tilt. The difference between the coupling degree on the two causal directions resulted as statistically significant at rest.

The traditional coherence evaluated at LF was significant (i.e., exceeded the zero-level threshold) in all subjects in both rest and tilt conditions. Differently, the number of subjects showing a significant causal coupling was 1 at rest and 9 after tilt for the feedback direction, and 10 at rest and 7 after tilt for the feedforward direction.

Fig. 3 reports the gain of the baroreflex transfer function estimated by noncausal and causal approaches on the healthy subject for which the coherence is reported in Fig. 1. While in the resting supine position the two LF estimates are markedly different, after tilt the values of causal and noncausal gain are fairly close.

(a)

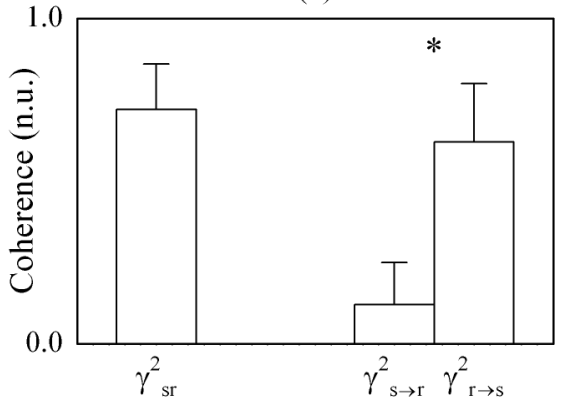

(b)

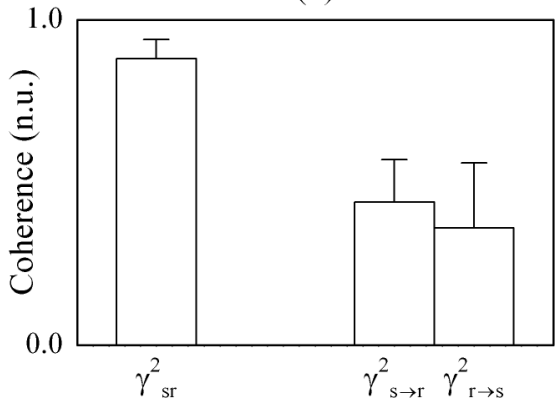

Figure 2. Mean (bars) + SD (whiskers) of the low frequency values of traditional $\left(\gamma_{s r}^{2}\right)$ and causal $\left(\gamma_{s \rightarrow r}^{2}\right.$ and $\gamma_{r \rightarrow s}^{2}$ ) coherences estimated with subjects in the supine position (a) and after head-up tilt (b). $* P<0.05$ (Student's $t$ test for paired data). (a)

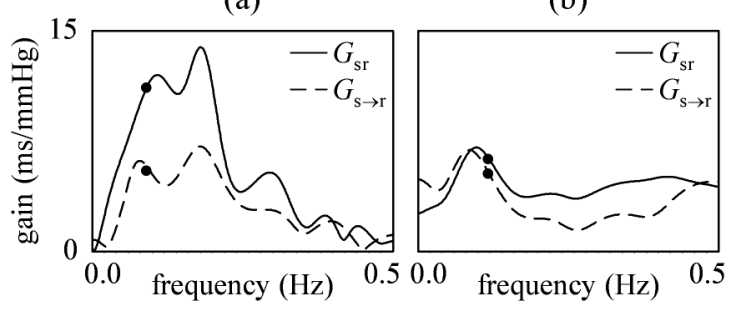

Figure 3. Example of calculation of the gain of the baroreflex transfer function by the noncausal approach (continuous line) and by the causal one (dashed line) for a subject in the supine (a) and in the upright (b) position. Filled circles indicate the functions sampled at the maximum coupling inside the low frequency band.

As shown in Fig. 4, the two approaches used to estimate the baroreflex gain from the cross-spectral functions produced statistically different values at rest, while the distributions of causal and noncausal gain estimates were substantially super imposable after tilt. 
(a)
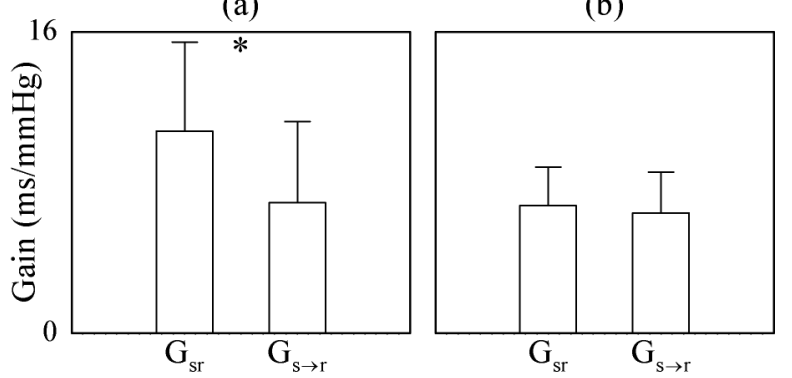

Figure 4. Mean (bars) + SD (whiskers) of the low frequency values of traditional $\left(G_{s r}\right)$ and causal $\left(G_{s \rightarrow r}\right)$ baroreflex gain estimated with subjects in the supine position (a) and after head-up tilt (b). $* P<0.05$ (Student's $t$ test for paired data).

\section{Discussion and conclusions}

The main result of the present study is that the widely studied baroreflex regulation of heart rate is not the only mechanism responsible for the interactions between RR and SP in healthy humans. Indeed, we found that a nonnegligible degree of coupling occurs in the causal direction from RR to SP at the frequency of the Mayer waves (around $0.1 \mathrm{~Hz}$ ). Particularly, such non-baroreflex interactions seem to play a major role in the determination of the RR-SP coupling when subjects lie in the supine position. With the orthostatic stimulation induced by tilt, the coupling on the two arms of the RRSP regulatory loop became balanced as a result of a significant baroreflex activation along with a slight weakening of the non-baroreflex interactions.

The comparison between the traditional cross-spectral estimation of the transfer function gain [2] and the corresponding causal approach [5] allowed us to assess the impact of non-baroreflex interactions in the spontaneous estimates of the baroreflex gain. As expected the causal estimate resulted always lower than the traditional one, and the difference was significant at rest, in accordance with the large presence of non-baroreflex interactions which are likely to corrupt the noncausal estimate [5].

Also the selection of the gain values deserves some comments. Commonly, a criterion based on the presence of a significant coherence is used to assess the reliability of transfer function values [9]. Despite this, in the present study the gain estimates were taken into consideration even when the coherence (or the causal coherence) was below the zero-level threshold determined as in [8]. The choice of relaxing the coherence criterion is due to (i) the consideration that non-significant coherence values only imply that the input-output relationship under analysis is negligible with respect to other sources affecting the output, but not that such a relationship cannot be investigated; (ii) the fact that low coherence values are often the expression of low system gain [9]; and (iii) the finding that the zero coherence test may often fail in detecting weak coupling degrees (i.e., the estimated coherence may often fall below the threshold even if the true coherence is nonzero) [8].

In conclusion, this study evidenced the necessity of accounting for causality in the analysis of the RR-SP interactions in physiological conditions. The utilization of causal cross-spectral analysis allowed us to detect the presence of significant non-baroreflex interactions, and to weigh their effects in the estimation of the spontaneous baroreflex gain.

\section{References}

[1] De Boer RW, Karemaker JM, Strackee J. Relationships between short-term blood-pressure fluctuations and heartrate variability in resting subjects: a spectral analysis approach. Med Biol Eng Comput 1985;23:352-8.

[2] Saul JP, Berger RD, Albrecht P, Stein SP, Hui Chen M, Cohen RJ. Transfer function analysis of the circulation: unique insights into cardiovascular regulation. Am J Physiol Heart Circ Physiol 1991;261:H1231-45.

[3] Malliani A. Principles of cardiovascular neural regulation in health and disease. Norwell (MA): Kluwer academic publishers, 2000.

[4] Porta A, Furlan R, Rimoldi O, Pagani M, Malliani A, van de Borne P. Quantifying the strength of the linear causal coupling in closed loop interacting cardiovascular variability signals. Biol Cybern 2002;86:241-51.

[5] Faes L, Porta A, Cucino R, Cerutti S, Antolini R, Nollo G. Causal transfer function analysis to describe the closed loop interactions between cardiovascular and cardiorespiratory variability signals. Biol Cybern 2004;90:390-9.

[6] Baselli G, Porta A, Rimoldi O, Pagani M, Cerutti S. Spectral decomposition in multichannel recordings based on multivariate parametric identification. IEEE Trans Biomed Eng 1997;44:1092-101.

[7] Akaike H. A new look at the statistical model identification. IEEE Trans Autom Contr 1974;19:716-23.

[8] Faes L, Pinna GD, Porta A, Maestri R, Nollo G. Surrogate data analysis for assessing the significance of the coherence function. IEEE Trans Biomed Eng 2004;51:1156-66.

[9] Pinna GD, Maestri R, Raczak G, La Rovere MT. Measuring baroreflex sensitivity from the gain function between arterial pressure and heart rate. Clin Sci 2002; 103:81-8.

Address for correspondence.

Luca Faes

Dept. of Physics, University of Trento

Via Sommarive 14, 38050 Povo (TN), Italy

E-mail address: faes@science.unitn.it 